\title{
Horizons/Théâtre
}

Revue d'études théâtrales

$14 \mid 2019$

Les arts du spectacle dans l'Afrique subsaharienne - 2

\section{Entretien avec Sèdjro Giovanni Houansou}

\section{Marjorie Bertin}

\section{(2) OpenEdition}

Journals

Édition électronique

URL : https://journals.openedition.org/ht/1765

DOI : $10.4000 /$ ht. 1765

ISSN : 2678-5420

\section{Éditeur}

Presses universitaires de Bordeaux

\section{Édition imprimée}

Date de publication : 1 janvier 2019

Pagination : 142-145

ISBN : 979-10-300-0718-3

ISSN : 2261-4591

\section{Référence électronique}

Marjorie Bertin, « Entretien avec Sèdjro Giovanni Houansou », Horizons/Théâtre [En ligne], 14 | 2019, mis en ligne le 01 juin 2022, consulté le 30 juin 2022. URL : http://journals.openedition.org/ht/1765 ; DOI : https://doi.org/10.4000/ht.1765

La revue Horizons/Théâtre est mise à disposition selon les termes de la Licence Creative Commons Attribution - Pas d'Utilisation Commerciale - Pas de Modification 4.0 International. 


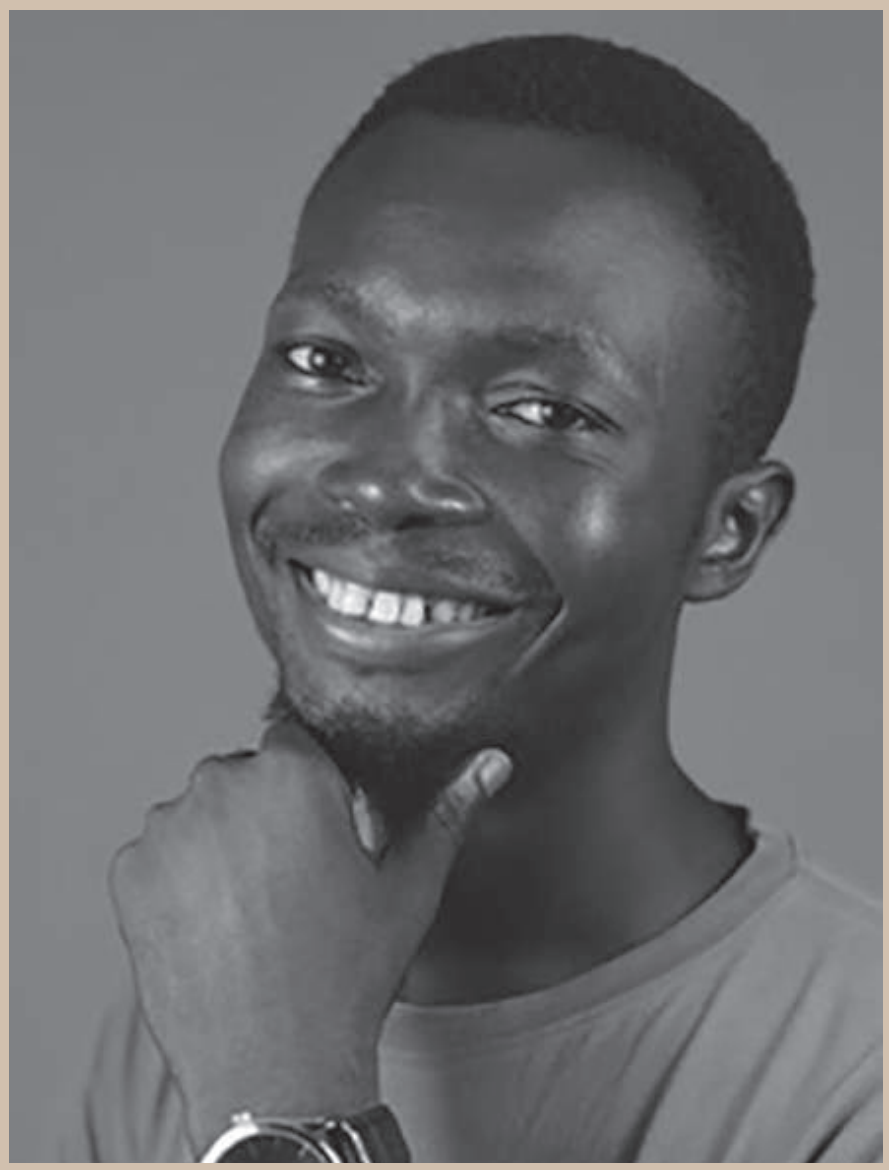

Né à Cotonou en 1987, Sèdjro Giovanni Houansou est auteur, metteur en scène et opérateur culturel béninois. Son travail artistique tourne beaucoup autour de la relation entre l'homme (l'humain) et le territoire.

En 2016, lauréat de Textes en scènes, il est accueilli en résidence au CNES - La Chartreuse de Villeneuve-lès-Avignon en 2016, pour écrire son troisième texte, La rue bleue après Sept milliards de voisins (2012) et Courses au Soleil (2014). En 2018 il bénéficie du dispositif Visas pour la création de l'Institut Français et son texte Les Inamovibles, qui explore des zones moins visibles des migrations SudNord, est lauréat du Prix RFI Théâtre 2018, et de l'aide à la création ARTCENA. Avec La rue bleve, il remporte le Grand Prix de la Littérature béninoise 2019. La même année, il est accueilli à la Maison des Auteurs de Limoges et à la Cité Internationale des Arts (Paris) pour écrire Les envahi.e.s mis en lecture au Théâtre des quartiers d'Ivry. Depuis 2012, Sèdjro Giovanni Houansou organise Les Embuscades de la Scène et Les didascalies du monde. II met en place en 2018 Échafaudages, premier studio ovest-africain des écritures dramatiques Jeunesse et la plate-forme benincrea.net. 


\section{Entretien avec Sèdjro Giovanni Houansou}

\section{Comment êtes-vous arrivé au théâtre ?}

J'ai commencé au Bénin au collège, puis au lycée en tant que comédien. Les textes sur lesquels on travaillait n'étaient pas, pour l'essentiel des pièces de théâtre, plutôt des adaptations de romans comme Sous l'orage de Seydou Badian. J'ai continué avec l'ensemble artistique et culturel des étudiants, et c'est en seconde que j'ai eu l'audace d'écrire une première pièce de théâtre, avec tout ce que cela pouvait comporter comme erreurs ! Elle s'appelait C'est dommage et traitait des promesses de campagne que les politiciens béninois faisaient pour gagner des voix et se faire élire.

Vous êtes passionné de problématiques très fortes, rarement traitées au théâtre, comme l'urbanisation sauvage dans La Rue bleue (2016). Comment ce texte a-t-il été reçu par les spectateurs béninois ?

Ils ont été surpris. Plus encore avec ma dernière pièce, Il pleut des humains sur nos pavés que je viens de présenter dans le cadre de La nuit des idées à l'Institut français de Lomé au Togo. Je suis d'un pays où la tradition théâtrale est très différente de ce qu'elle est en Europe, particulièrement en France. Les gens ne sont pas habitués aux sous-textes, aux doubles sens - ce que je pratique dans mon écriture est très nouveau pour eux. Ils sont souvent surpris et intéressés. C'est le cas aussi bien sûr avec les auteurs francophones qui viennent aux Didascalies du monde, où nous organisons toujours, à l'issue de la lecture de la pièce théâtralisée, une rencontre en bord de plateau avec l'auteur. Il y a quelque temps, un auteur français, Romain Nicolas est venu présenter une pièce qui traite du développement du nucléaire et de ses effets nocifs pour l'environnement. C'était quelque chose de très nouveau pour les Béninois. On ne parle jamais de ce genre de sujet ici. Autre exemple : la fabrication de certains médicaments est très, nocive très nocive pour l'environnement. Les spectateurs étaient très étonnés mais très intéressés de voir ces réflexions dans un texte de théâtre. Les gens me disent qu'ils en ont besoin. 


\section{Est-ce pour eux que vous écrivez essentiellement ?}

Non. Aucun sujet n'est absolument universel bien sûr mais j'essaie de toucher le maximum de gens possible et notamment en France. Cela dit il y a un vrai tiraillement pour les auteurs d'Afrique francophone entre la réception en France et en Afrique. La première pièce que j'ai écrite (en tant que professionnel) 7 milliards de voisins a été très bien reçue en Afrique de l'Ouest mais n'a eu aucun succès dans les comités de lecture francophones. J'ai écrit ensuite Course au soleil et là j'ai eu de très bons retours des comités de lecture francophones mais cela ne fonctionnait pas au Bénin!

\section{Comment expliquez-vous ce phénomène ?}

La langue de 7 milliards de voisins était trop crue, trop directe pour les comités de lecture en France. Certains lecteurs ont trouvé que c'était un texte creux, qui manquait de traits d'esprit. Il y a un réel décalage entre l'écriture de théâtre en France et au Bénin, je m’en suis aperçu en allant en Résidence à la Chartreuse des Avignon en 2016. Il y avait de jeunes auteurs d'un peu partout et notamment de grandes écoles comme l'ENSAT. Au cours de nos échanges, j'étais bouleversé de voir que pour moi, ce qu'ils faisaient et proposaient n'appartenait pas au théâtre, les problématiques de recherche, les esthétiques étaient très différentes de celles du théâtre béninois. Le Bénin n'est pas globalement en retard par rapport à un pays comme la France mais le théâtre béninois est très en marge des recherches et des expérimentations européennes.

Est-ce que le détour par la France reste un passage important pour réussir lorsque l'on veut être reconnu sur le Continent ?

Non mais il est nécessaire pour un auteur qui veut faire son chemin en France d'y venir pour voir le théâtre qui s'y fait, pour rencontrer d'autres structures, d'autres esthétiques, d'autres dramaturgies que ce qui existe au Bénin. C'est nécessaire et c'est pour cette raison que nous avons créé la plateforme les Didascalies du monde à Cotonou, en 2018 afin que les artistes béninois puissent rencontrer d'autres écritures et aussi des structures qui accueillent les créations.

\section{De quoi s'agit-il exactement ?}

C'est une plate-forme qui accueille chaque année, dix textes de théâtre francophones qui sont mis en lecture dans différents espaces, publics ou privés. Ils sont sélectionnés par un appel à candidatures, puis l'on propose 
à des metteurs en scène de s'en emparer. On met en rapport ces metteurs en scène avec de jeunes comédiens avec lesquels ils répètent pour faire ensuite entendre chaque texte. Cela s'étend sur dix mois de l'année, à raison d'un texte par mois. Ce sont plus des lectures que des créations théâtrales, mais les metteurs en scène ont la liberté de donner aux comédiens des indications de jeu, par exemple. L'idée est vraiment de faire entendre ces textes, qui viennent du Bénin, de toute l'Afrique de l'Ouest mais aussi de la France, et d'organiser, à l'issue de la lecture, un bord de scène. Aujourd' hui nous sommes en train de travailler pour obtenir du matériel de sonorisation et des pupitres afin de pouvoir sortir des salles. Nous avons également mis en place d'autres platesformes : Échafaudage se tient dans les collèges et les lycées, j'essaie avec ce dispositif de reproduire ce que j'ai vécu en résidence à la Chartreuse, mais l'idée n'est pas de former des futurs auteurs de théâtre. Ce que l'on essaie de faire surtout, pendant une semaine c'est d'essayer d'intéresser ces jeunes, de seize et vingt-et-un ans à l'écriture dramatique. Nous avons également créé un site internet, Benincrea.net, qui rassemble les appels à candidatures pour des festivals ou des résidences qui sont en Afrique ou ouverts à des artistes du Continent. C'était important d'essayer de les mettre à disposition sur un site, car ce sont des informations qui sont rarement accessibles au Bénin. En 2013, nous avons également créé une autre plate-forme, qui s'appelle Les Embuscades de la scène. L'idée est de donner leur première chance à des jeunes metteurs en scène. On met à leur disposition un cadre de travail avec un metteur en scène professionnel qui les guide et on cherche de notre côté des structures pour qu'ils puissent être joués une première fois. Cela les aide à se faire un nom. Sans recommandation les jeunes n'arrivent pas être programmés dans un Institut français ou Artisttik Africa (centre culturel africain de Cotonou, $n d l r$ ).

Malheureusement, comme je n'ai pas encore de salle c'est un projet très coûteux qui est mis en pause actuellement. Enfin et surtout nous sommes en pleine création de l'Artiwrium, une plate-forme pour rendre accessible et possible la formation artistique, à travers des vidéos, des webinars et des tutoriels. L'idée est à la fois de valoriser le patrimoine culturel de la pratique théâtrale africaine, de transmettre des savoirs et des pratiques, mais aussi de faire des tutoriels pour enseigner des techniques artistiques concrètes, comme par exemple comment obtenir telle couleur sur une toile, etc. On tâche enfin aussi de former les gens au management de l'art et de la culture en partenariat avec les universités. C'est passionnant et très ambitieux!

Par Marjorie Bertin 